\title{
Seven Common Allergen Groups Causing Eyelid Dermatitis: Education and Avoidance Strategies
}

This article was published in the following Dove Press journal:

Clinical Ophthalmology

\author{
Crystal X Huang (D) \\ James A Yiannias ${ }^{2}$ \\ Jill M Killian ${ }^{3}$ \\ Joanne F Shen $\mathbb{D D}^{4}$
}

'Mayo Clinic Alix School of Medicine, Scottsdale, AZ, USA; ${ }^{2}$ Department of Dermatology, Mayo Clinic, Scottsdale, AZ, USA; ${ }^{3}$ Department of Health Sciences Research, Mayo Clinic, Rochester, MN, USA; ${ }^{4}$ Department of Ophthalmology, Mayo Clinic, Scottsdale, AZ, USA
Correspondence: Joanne F Shen

Tel + I 480-30I-8085

Fax + I 480-30I-7326

Email Shen.Joanne@mayo.edu
Objective: Eyelid dermatitis is most commonly attributed to allergic response. This retrospective clinical study identifies common allergens with eyelid involvement and addresses a literary gap by providing a clear approach for effective management of periorbital allergic contact dermatitis (ACD) recurrence.

Methods: Charts of 215 patients diagnosed with periorbital dermatitis who were patch tested with Mayo Clinic Standard Series, Extended Standard Series, and personal products from 2013 to 2017 were examined. Positive reaction rates for patients with eyelid involvement were compared to those without. Findings were also compared to North American Contact Dermatitis Group (NACDG) 2013-2014 and Mayo Clinic Contact Dermatitis Group (MCCDG) 2011-2015 general patch test populations.

Results: The 215 patients showed more common allergy to shellac, benzalkonium chloride, acrylates, and surfactants than the NACDG and MCCDG study populations. Periorbital ACD allergen groups eliciting the highest positive reaction rates were, in descending order: metals, shellac, preservatives, topical antibiotics, fragrances, acrylates, and surfactants. Of the corticosteroids, only tixocortol pivalate (the screening agent for prednisolone and fluorometholone) and budesonide elicited positive reactions.

Conclusion: The top seven eyelid ACD allergen groups were identified. Avoidance of these allergens can be straightforward, with initial empiric counseling and free, online allergen avoidance programs. Patients who are unresponsive to avoidance should undergo patch testing.

Keywords: allergic contact dermatitis, periorbital skin, patch test, contact allergens, allergen avoidance program

\section{Introduction}

Allergic contact dermatitis (ACD) is a delayed type IV hypersensitivity reaction, peaking 24-48 hours after allergen presentation. ${ }^{1}$ Acute ACD symptoms include vesicles or papules, macules, erythema, and periorbital swelling; chronic ACD symptoms include fissuring, lichenification, and scaling. ${ }^{2,3}$ The differential diagnosis for ACD includes irritant contact dermatitis, seborrheic dermatitis, atopic dermatitis, phototoxic dermatitis, psoriasis, rosacea, urticaria, dermatomyositis, cutaneous T-cell lymphoma, blepharitis, and other infections. ${ }^{4-7}$

Particular susceptibility of the eyelids to ACD has been widely reported. ${ }^{6-12}$ Multiple factors contribute to this observation: the eyelids constitute the thinnest skin on the body $(0.55 \mathrm{~mm})$, thus allowing for easier allergen penetration; their unprotected position on the body invites substantial cosmetic and environmental exposure, including frequent contact with allergens carried by the hands; and they 
function as a regular application site of many eye care medications and products. ${ }^{7-9,11}$ In fact, the eyelids may manifest as the initial or singular site of ACD symptoms, even with allergen exposure occurring elsewhere on the body. $^{6,7,12}$ Consequently, ophthalmologists and optometrists are often the first providers to encounter patients with ACD.

Identification of $\mathrm{ACD}$ as the most frequent cause of eyelid dermatitis has been well established, especially in the dermatologic literature. ${ }^{4,8,12-15}$ Eye care providers are quick to point to topical ophthalmic medications as potential ACD triggers. However, ACD is often misdiagnosed, or ophthalmologists and optometrists may be unsure of therapeutic options if patients fail to improve despite avoidance of ophthalmic medications. ${ }^{3,16}$ When confronted with ACD, dermatologists often recommend patch testing, which traditionally involves the application of potential allergenic substances at dilute concentrations to the patient's skin via "patches," worn for 48 hours, with test interpretation at 96 hours. $^{2}$

Patch testing, widely regarded as the gold standard for ACD diagnosis, is deemed a necessity in ACD treatment because accurate identification and proper avoidance of responsible allergen(s) offer a simple, effective cure. ${ }^{17-19}$ Allergens in standard patch test series are recommended to have clinical relevance and/or positive reaction rates greater than $0.5-1.0 \%{ }^{20}$ Citing patch test results, previous case series and reviews have highlighted allergens in such groups as preservatives, cosmetics, anti-inflammatory medications, anesthetics, and anti-glaucoma medications. ${ }^{1,3}$

Literary gaps exist, especially in ophthalmologic journals, on the causes of isolated periorbital ACD, and on modern clinical approaches to prevent ACD recurrence following allergen identification. . $3,8,11,21,22$ Moreover, if patch testing is unavailable, other strategies must be employed. This retrospective clinical study identifies ACDrelated common allergens with eyelid involvement, compares current findings to previous reports, and advances the current literature by offering a straightforward, contemporary approach to identify patient products with and without common offending allergens for periorbital ACD management.

\section{Methods}

This retrospective study followed the ethical tenets of the Declaration of Helsinki and underwent Mayo Clinic Institutional Review Board (IRB) approval. The study was found to constitute minimal risk research, with an approved waiver of informed consent and adequate precautions taken to de-identify subjects' information and protect patient data confidentiality. From January 1, 2013 through December 31, 2017, 217 patients diagnosed with dermatitis involving the eyelids and 1975 patients with dermatitis not involving the eyelids were patch tested at Mayo Clinic, Arizona, to our Standard Series and Extended Standard Series (151 allergens). All patients were diagnosed with any of the following: asteatotic dermatitis, atopic dermatitis, allergic dermatitis, irritant dermatitis, dermatitis not otherwise specified, and/or seborrheic dermatitis.

Patch testing followed North American Contact Dermatitis Group (NACDG) standard guidelines (eg no oral or topical corticosteroid involvement; reactions graded on a standardized scale). ${ }^{20}$ Patches were removed 48 hours after application to patients' upper backs. Reaction sites were evaluated, first immediately after patch removal, then again 92-108 hours after removal (as shown in Figure 1). The Mayo Clinic Standard Series included contact lens materials (eg methyl acrylates), antibiotics (eg neomycin),

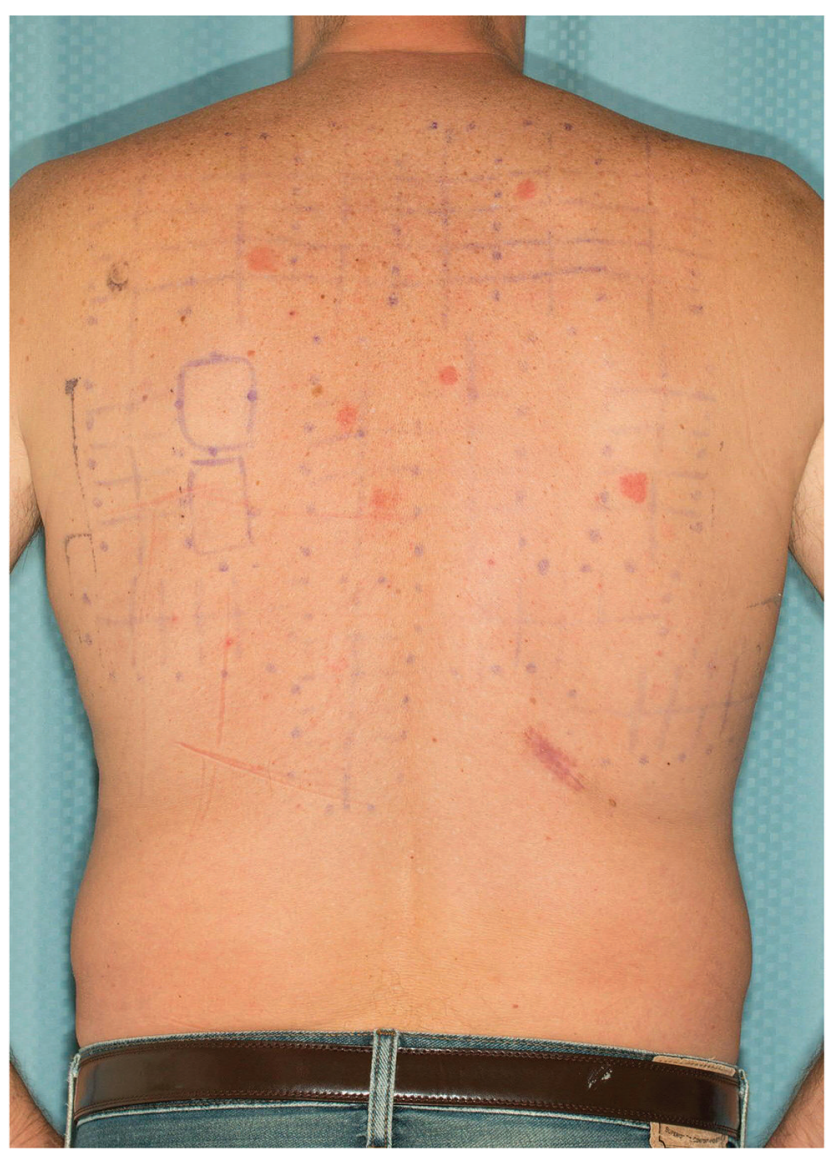

Figure I Patient showing positive reactions at several application sites of distinct allergens, following the removal of patches. 
anesthetics (eg benzocaine, lidocaine), and corticosteroids (eg hydrocortisone-17-butyrate, tixocortol pivalate, clobetasol-17-butyrate, triamcinolone, budesonide, desoximetasone), while the Mayo Clinic Extended Standard Series included such known allergens as thimerosal. (Of note for ophthalmologists, steroids in Group 1 [eg prednisolone, tixocortol pivalate, budesonide, loteprednol, fluorometholone, and difluprednate] and Group 2 are the most likely to cause contact allergy, while steroids in Group 3 [eg rimexolone, dexamethasone] are the least likely; Table 1 displays additional examples in each group. ${ }^{23,24}$ ) More importantly, the Mayo Clinic Standard Series includes several allergens frequently found in skincare products that are not included in the NACDG Standard Series, for potential clinical relevance in spite of relatively low positive reaction rates. $^{25}$

Allergen test results were defined as follows based on day 5 readings: positive, if day 5 reaction grade $=2$, 3 or 4 ; negative, if day 5 reaction grade $=0$ or 1 ; or irritant, if reaction relevancy $=\mathrm{I}$. Reaction grade was defined as follows: $0=$ negative; $1=$ macular erythema; 2 = weak (non-vesicular erythema, infiltration or papules); $3=$ strong (edema or vesicles); $4=$ extreme (bullous, ulcerative or spreading lesions). ${ }^{20}$ Reaction relevancy was defined as follows: $\mathrm{I}=$ irritant; $\mathrm{N}=$ allergic, not relevant; $\mathrm{P}=$ allergic, formerly relevant; $\mathrm{Q}=$ allergic, questionably relevant; $\mathrm{R}=$ allergic, relevant.

Since two patients with eyelid involvement did not have reports of day 5 reaction results, a total of 215

Table I Classification of Topical Corticosteroids Used in Ophthalmic Preparations

\begin{tabular}{|l|l|l|}
\hline Group I & Group 2 & Group 3 \\
\hline Class A, D2 Plus Budesonide & Class B & Class C, DI \\
\hline $\begin{array}{l}\text { Testing agent: } \\
\text { Tixocortol pivalate }\end{array}$ & $\begin{array}{l}\text { Testing agent: } \\
\text { Triamcinolone }\end{array}$ & $\begin{array}{l}\text { Testing agent: } \\
\text { Desoximetasone }\end{array}$ \\
\hline $\begin{array}{l}\text { Difluprednate (Durezol) } \\
\text { Fludrocortisone acetate } \\
\text { (Flarex) }\end{array}$ & Triamcinolone & $\begin{array}{l}\text { Clobetasol } \\
\text { Dexamethasone } \\
\text { Fluorometholone (FML) } \\
\text { (Maxidex) } \\
\text { Hydrocortisone acetate } \\
\text { Prednisolone acetate (Pred } \\
\text { Mild, Pred Forte, } \\
\text { Blephamide) } \\
\text { Prednisolone sodium } \\
\text { phosphate }\end{array}$ \\
\hline
\end{tabular}

patients with eyelid involvement were included in analysis. For these patients, reaction rates to their own products (365 patient products) were examined as well.

Patient characteristics (basic demographic information, ie age, sex, race), results by allergen sorted by descending number of patients tested, and results by allergen sorted by descending positivity were recorded and examined.

Positive reaction rates for patients with eyelid involvement were compared to those for patients without eyelid involvement. Findings were also compared to general patch test patient populations as reported by the North American Contact Dermatitis Group (NACDG) on 4871 patients tested from 2013 to 2014, and to those reported by the Mayo Clinic Contact Dermatitis Group (MCCDG) Standard Series on 2582 patients tested from 2011 to 2015 .

\section{Results}

Of the 215 patients with eyelid dermatitis (mean [SD] age, 53.1 [15.0] years) during the 4-year study period at Mayo Clinic in Arizona, $184(85.6 \%)$ were female and 31 (14.4\%) were male. These patients' reported racial demographics were as follows: $189(92.2 \%)$ White, 5 (2.4\%) Black, 4 (2.0\%) Asian, 1 (0.5\%) American Indian/Alaska Native, 6 (2.9\%) Other, and 10 unknown. Given these demographics, we acknowledge that these results may not be completely applicable to patients with skin of color.

Of the Standard and Extended Standard Series, 74 allergens elicited positive reactions in these 215 patients with eyelid involvement. Table 2 displays the reaction rates for these 74 allergens (sorted by descending positivity in the patient population with eyelid involvement), alongside corresponding reactions rates for these allergens in the 1975 patients without eyelid involvement, and in the general patch test populations NACDG 2013-2014 ${ }^{26}$ and MCCDG 2011-2015. ${ }^{20}$ Table 3 displays the 15 allergens eliciting the highest reaction rates in each of these four populations, ie Mayo Clinic 2013-2017 with eyelid involvement, Mayo Clinic 2013-2017 without eyelid involvement, NACDG 2013-2014, ${ }^{26}$ and MCCDG 2011-2015 $5^{20}$ (sorted by descending positivity).

For the 215 patients with eyelid involvement, the highest reaction rates were elicited by the following allergen groups, starting with the highest rate of positivity: metals (eg, nickel in eyewear, gold in eye makeup); shellac (a tackifier that helps skincare products adhere to the skin); preservatives (eg, benzalkonium chloride $[\mathrm{BAK}]$ in skincare products, prescription and over-the-counter eye preparations); topical antibiotics (neomycin, bacitracin); 


\begin{tabular}{|c|c|c|c|c|c|c|c|c|c|c|c|c|}
\hline \multicolumn{2}{|c|}{ 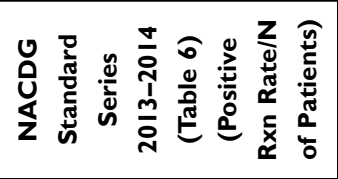 } & 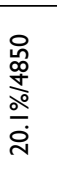 & 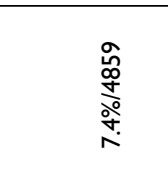 & 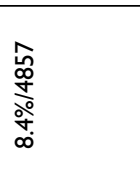 & & 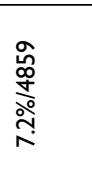 & 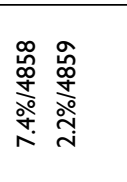 & & & 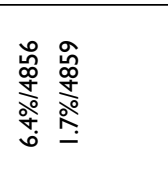 & 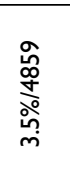 & 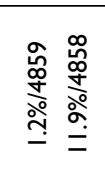 \\
\hline \multicolumn{2}{|c|}{ 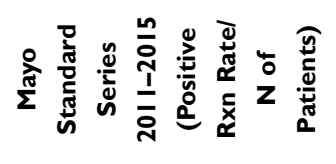 } & 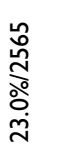 & 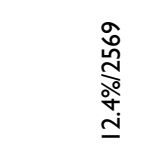 & 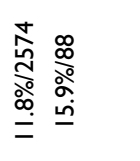 & 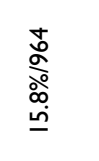 & 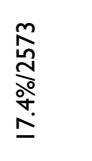 & 点 & & & 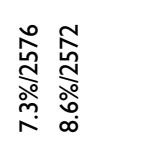 & & 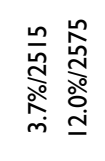 \\
\hline \multirow{5}{*}{ 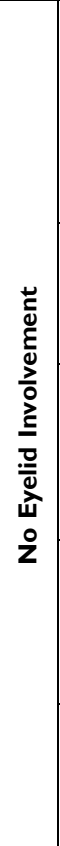 } & 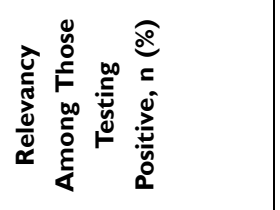 & 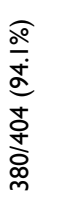 & 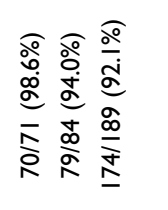 & 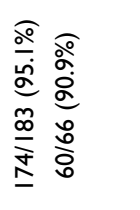 & 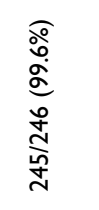 & 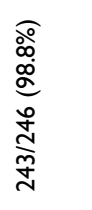 & 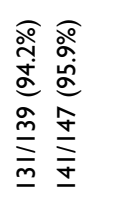 & 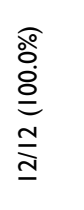 & 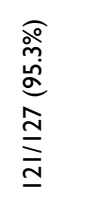 & 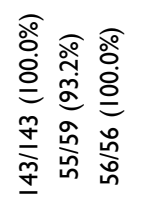 & 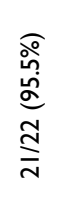 & 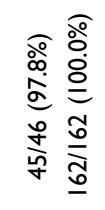 \\
\hline & 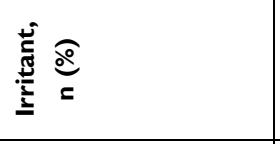 & $\begin{array}{l}\stackrel{\nwarrow}{\stackrel{一}{\leftrightarrows}} \\
\bar{N}\end{array}$ & 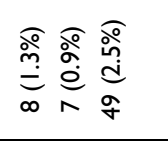 & 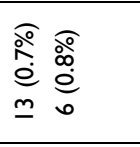 & 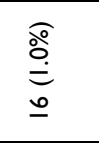 & 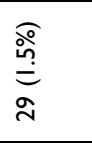 & 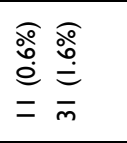 & 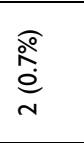 & $\begin{array}{l}\stackrel{\circ}{\circ} \\
\stackrel{\circ}{\circ} \\
=\end{array}$ & 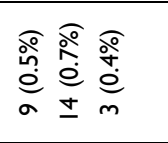 & 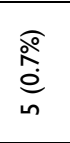 & 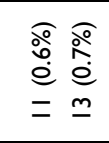 \\
\hline & 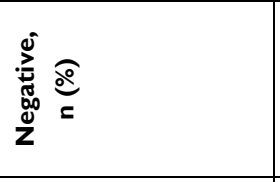 & 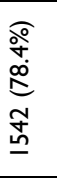 & 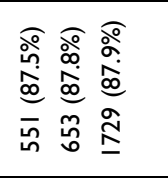 & 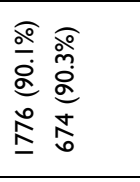 & 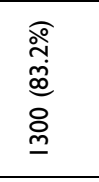 & $\begin{array}{l}\frac{\widehat{o}}{\dot{0}} \\
0 \\
\frac{0}{\hat{\sigma}} \\
\underline{0}\end{array}$ & 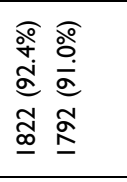 & 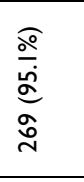 & 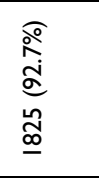 & 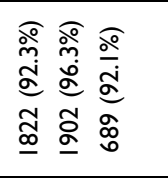 & $\begin{array}{l}\widehat{o} \\
\stackrel{d}{d} \\
\bar{d}\end{array}$ & 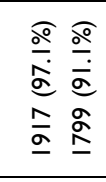 \\
\hline & $\sum_{\substack{0 \\
0}}^{0}$ & 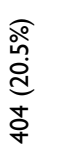 & 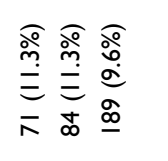 & 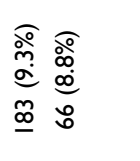 & 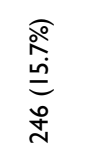 & 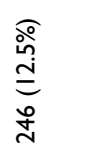 & 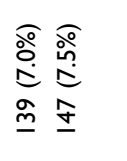 & 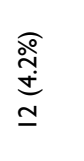 & $\begin{array}{l}\widehat{o} \\
\stackrel{+}{+} \\
\stackrel{0}{\Xi} \\
\text { I }\end{array}$ & 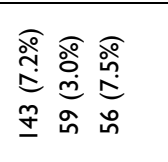 & 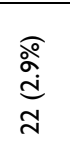 & 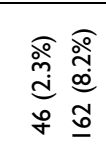 \\
\hline & 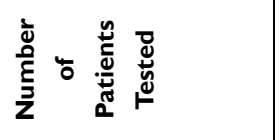 & ळ & 审素。 & $\underset{2}{\mathbb{2}}$ & 온 & $\underset{\sigma}{N}$ & 총 & $\stackrel{\sim}{\sim}$ & $\stackrel{\circ}{\circ}$ & 志占占 & $\stackrel{\infty}{\lessgtr}$ & 志志 \\
\hline \multirow{5}{*}{ 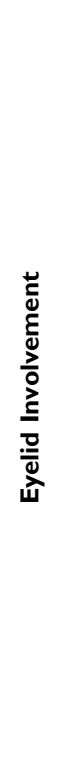 } & 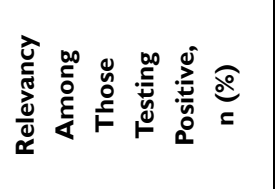 & $\begin{array}{l}\frac{0}{9} \\
\frac{a}{\sigma} \\
\frac{2}{0}\end{array}$ & 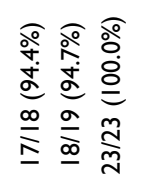 & 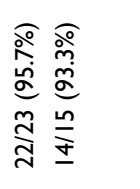 & 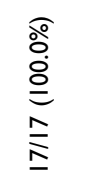 & 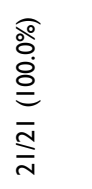 & 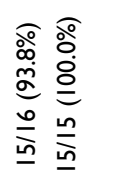 & 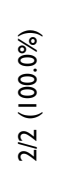 & $\begin{array}{l}\text { @ò. } \\
\stackrel{8}{0} \\
\text { o }\end{array}$ & 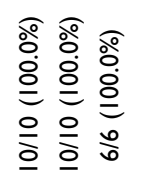 & $\begin{array}{l}\text { ळo } \\
\stackrel{0}{0} \\
\stackrel{0}{0} \\
\stackrel{0}{0}\end{array}$ & 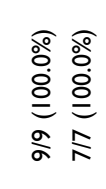 \\
\hline & $\stackrel{\overrightarrow{\mathrm{E}}}{\stackrel{\overrightarrow{\mathrm{E}}}{\mathrm{E}} \mathrm{o}}$ & 0 & 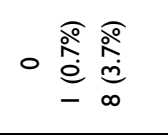 & 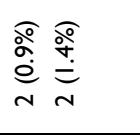 & $\begin{array}{l}\stackrel{\circ}{\circ} \\
\stackrel{0}{\stackrel{0}{0}}\end{array}$ & 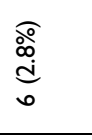 & 总 & 0 & 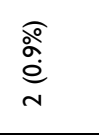 & 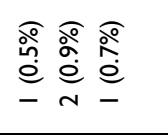 & $\frac{\sqrt{\frac{o}{d}}}{m}$ & 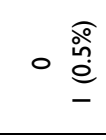 \\
\hline & 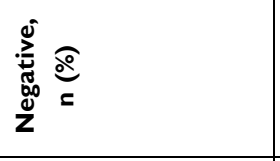 & $\begin{array}{l}\text { } \\
\stackrel{\circ}{\circ} \\
\stackrel{\infty}{0} \\
\underline{0}\end{array}$ & 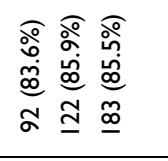 & 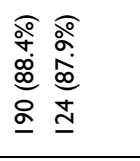 & 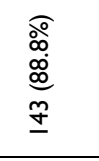 & 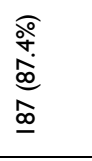 & 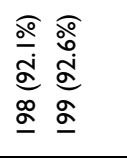 & 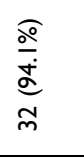 & 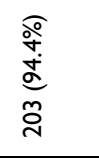 & 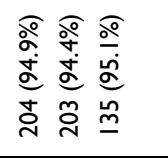 & $\begin{array}{l}\stackrel{\curvearrowright}{\circ} \\
\stackrel{p}{0} \\
\stackrel{m}{m}\end{array}$ & 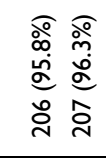 \\
\hline & $\stackrel{\substack{0 \\
0}}{0}$ & & 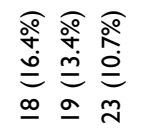 & 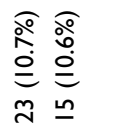 & 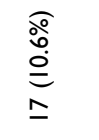 & 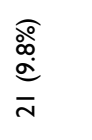 & 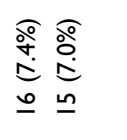 & 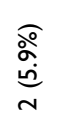 & 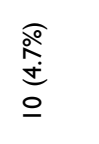 & 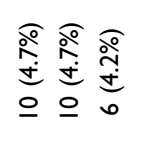 & 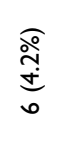 & 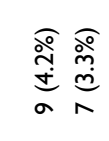 \\
\hline & 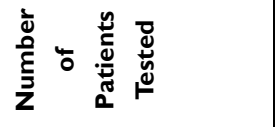 & $\frac{m}{N}$ & $\underline{q} \stackrel{\frac{\pi}{N}}{ }$ & $\stackrel{n}{N} \bar{\xi}$ & $\underline{\Phi}$ & $\stackrel{\nabla}{\sim}$ & $\frac{n}{N}$ & $\stackrel{+}{m}$ & $\stackrel{n}{\sim}$ & $\frac{n}{N} \frac{n}{N} \stackrel{q}{I}$ & 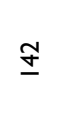 & $\stackrel{n}{N} \frac{n}{N}$ \\
\hline \multicolumn{2}{|l|}{ 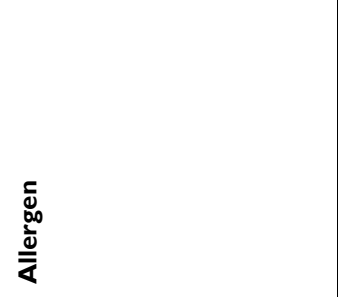 } & & 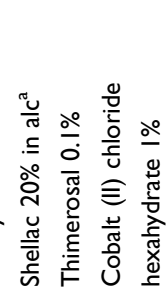 & 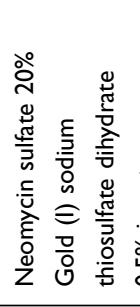 & 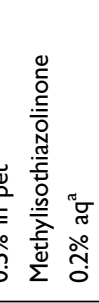 & 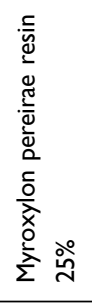 & 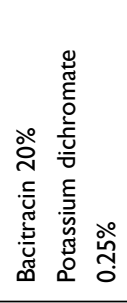 & 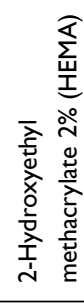 & 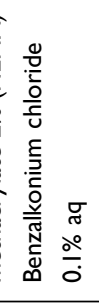 & 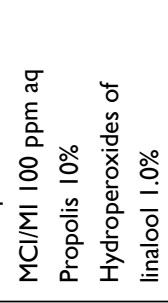 & 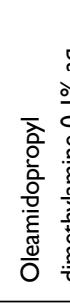 & 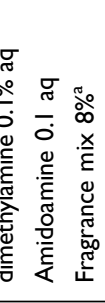 \\
\hline
\end{tabular}




\begin{tabular}{|c|c|c|c|c|c|c|c|}
\hline 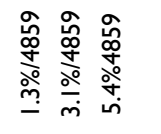 & & 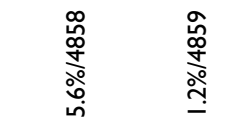 & & 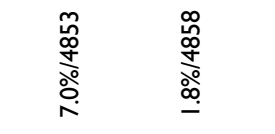 & 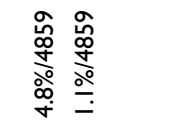 & 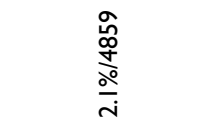 & 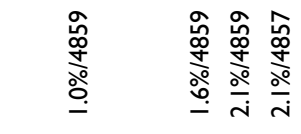 \\
\hline 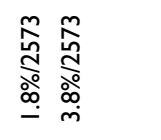 & 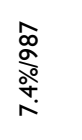 & 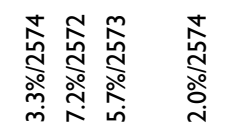 & 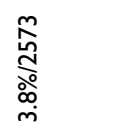 & 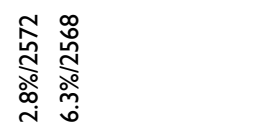 & 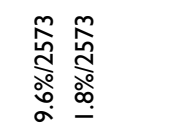 & 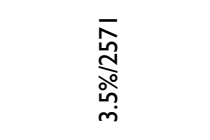 & 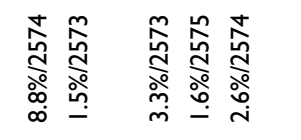 \\
\hline 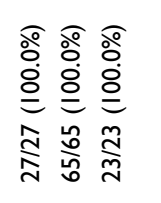 & 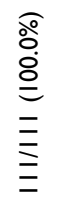 & 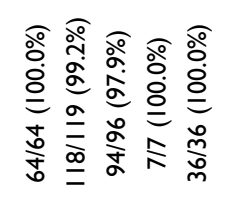 & 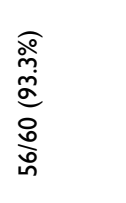 & 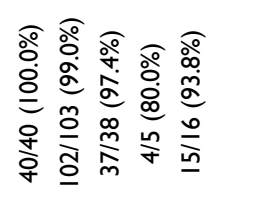 & 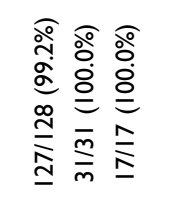 & 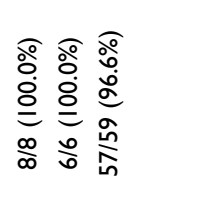 & 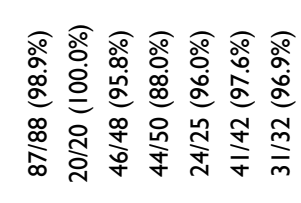 \\
\hline 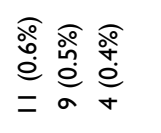 & $\begin{array}{l}\text { ơ } \\
\stackrel{0}{0} \\
\stackrel{0}{0}\end{array}$ & 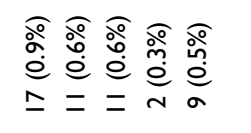 & 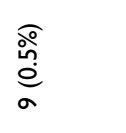 & 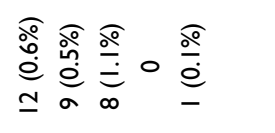 & 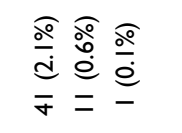 & 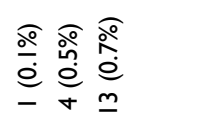 & 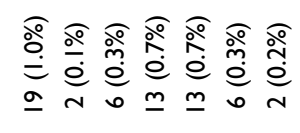 \\
\hline 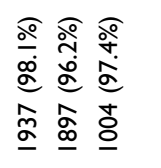 & 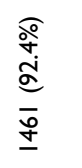 & 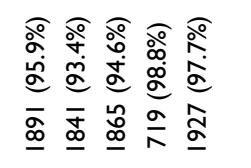 & 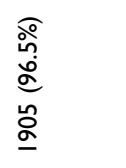 & 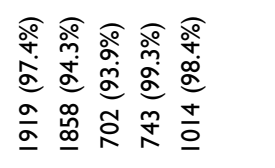 & 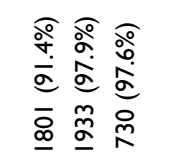 & 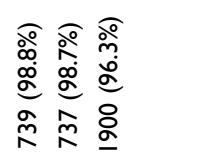 & 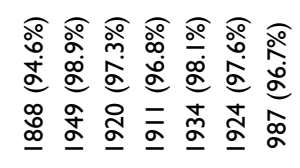 \\
\hline 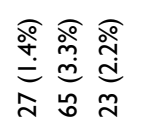 & 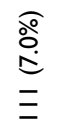 & 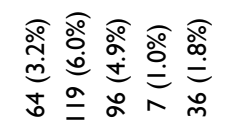 & $\begin{array}{l}\text { } \\
\stackrel{\circ}{0} \\
\stackrel{0}{\circ}\end{array}$ & 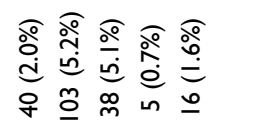 & 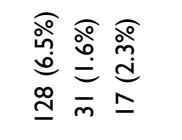 & 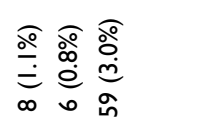 & 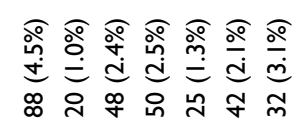 \\
\hline 占合㑒 & $\stackrel{\mathscr{D}}{\stackrel{0}{n}}$ & 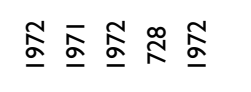 & $\underset{\Delta}{\stackrel{5}{a}}$ & 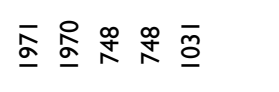 & 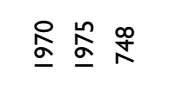 & 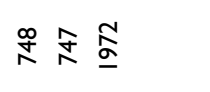 & 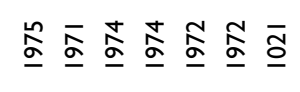 \\
\hline 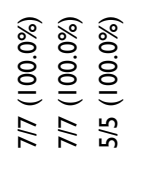 & 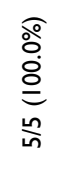 & 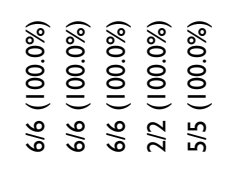 & 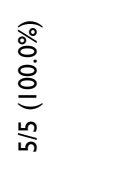 & 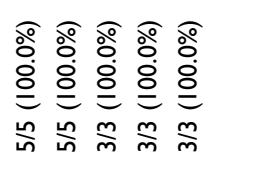 & 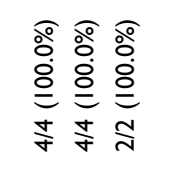 & 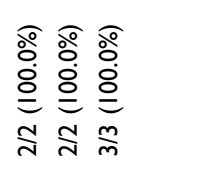 & 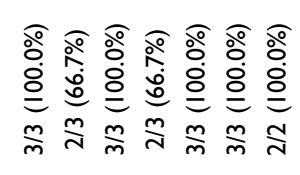 \\
\hline 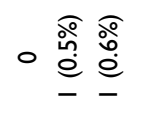 & $\underset{\sim}{\stackrel{\overbrace o}{ \pm}}$ & 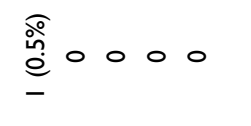 & 0 & 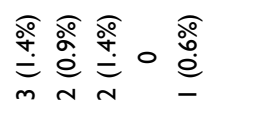 & 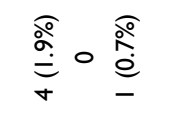 & 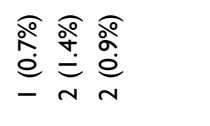 & 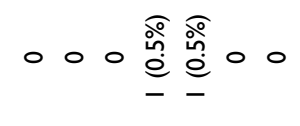 \\
\hline 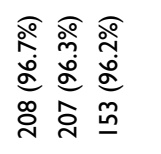 & 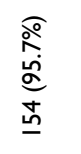 & 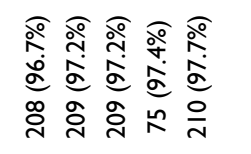 & 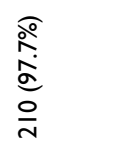 & 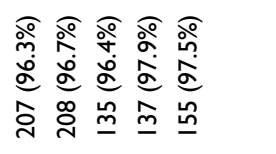 & 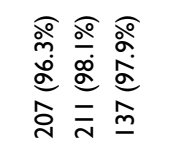 & 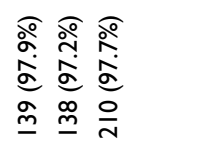 & 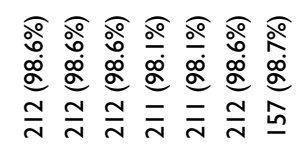 \\
\hline 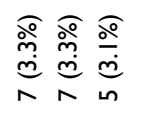 & 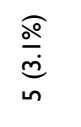 & 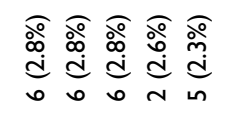 & 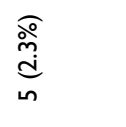 & 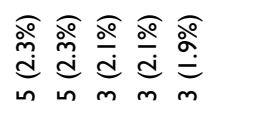 & 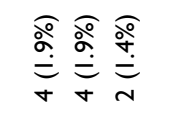 & 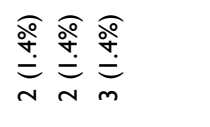 & 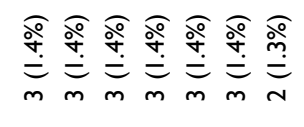 \\
\hline 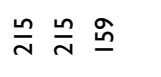 & $\underline{\overline{0}}$ & $\stackrel{n}{N} \frac{n}{N} \frac{n}{N} R \frac{n}{N}$ & $\stackrel{n}{N}$ & $\stackrel{n}{N} \frac{n}{N}$ 守守 总 & $\frac{n}{N} \frac{n}{N} \frac{Q}{9}$ & $\underline{q} \underline{\underline{q}} \frac{n}{N}$ & 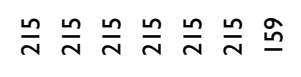 \\
\hline \multicolumn{8}{|c|}{ 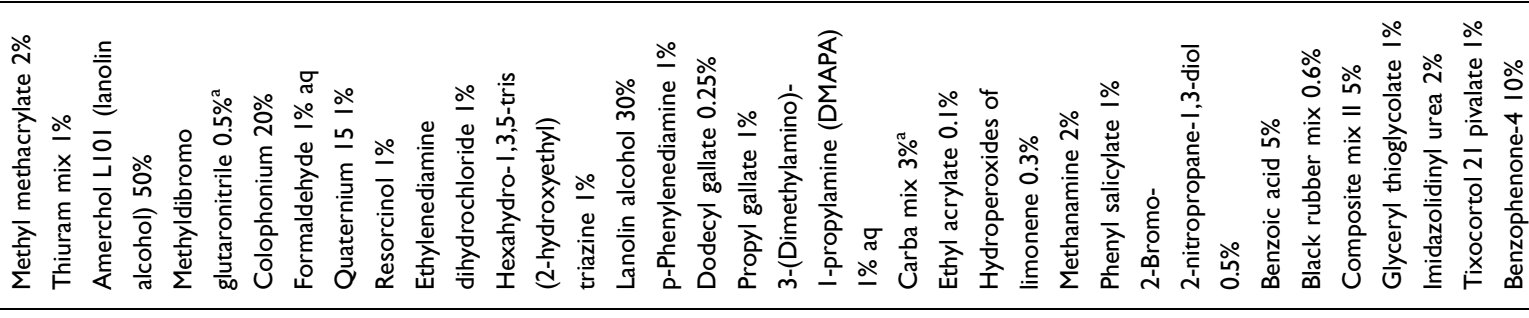 } \\
\hline
\end{tabular}




\begin{tabular}{|c|c|c|c|c|c|c|c|c|c|}
\hline \multicolumn{2}{|c|}{ 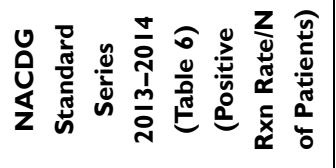 } & & 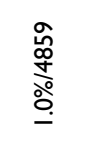 & 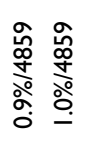 & 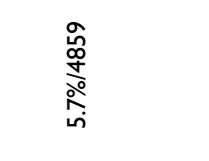 & 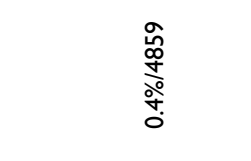 & 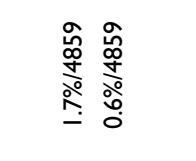 & 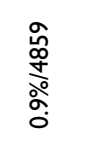 & 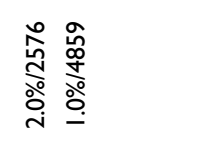 \\
\hline \multicolumn{2}{|c|}{ 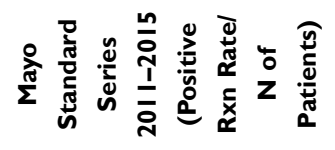 } & & 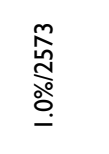 & 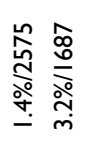 & 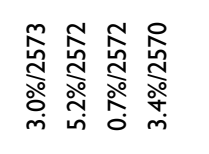 & & 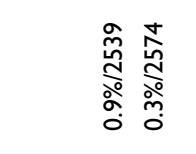 & 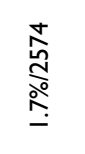 & 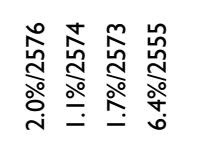 \\
\hline \multirow{5}{*}{ 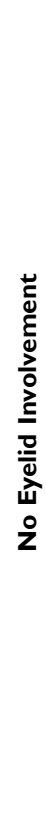 } & 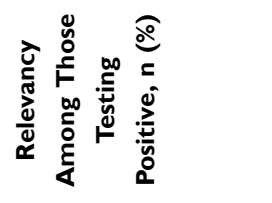 & $\begin{array}{l}\widehat{\circ} \\
\stackrel{\circ}{i} \\
\stackrel{5}{c} \\
\frac{1}{m}\end{array}$ & 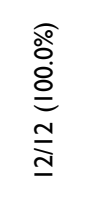 & 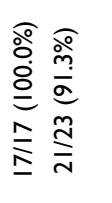 & 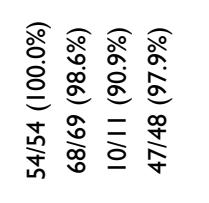 & 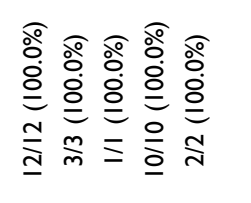 & 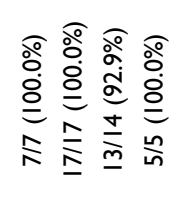 & $\begin{array}{l}\widehat{\circ} \\
\stackrel{\circ}{\circ} \\
\bar{\Xi} \\
\bar{N}\end{array}$ & 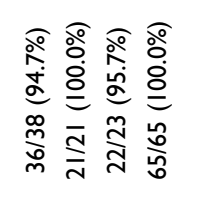 \\
\hline & 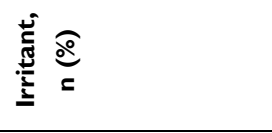 & 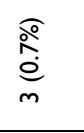 & $\underset{\substack{\stackrel{\circ}{d} \\
\stackrel{c}{c}}}{r}$ & 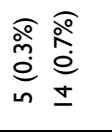 & 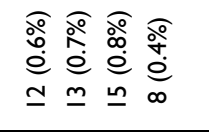 & 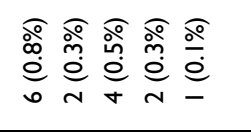 & 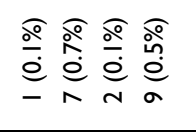 & $\begin{array}{l}\stackrel{\circ}{\circ} \\
\stackrel{\varrho}{\varrho} \\
\simeq\end{array}$ & 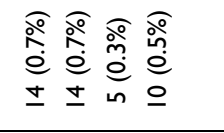 \\
\hline & 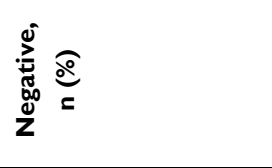 & 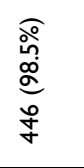 & 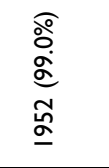 & 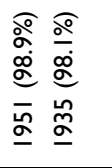 & 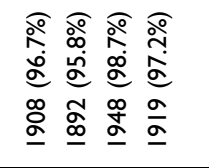 & 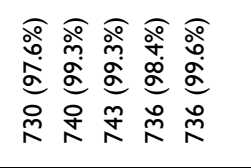 & 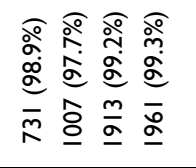 & $\begin{array}{l}\frac{\widehat{o}}{\infty} \\
\stackrel{\infty}{0} \\
\stackrel{\sigma}{0}\end{array}$ & 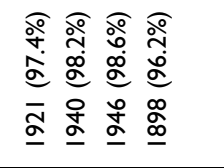 \\
\hline & $\stackrel{5}{\stackrel{0}{0}}$ & $\underset{\substack{\circ \\
\stackrel{\sigma}{\circ}}}{\stackrel{\sigma}{c}}$ & 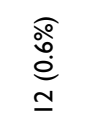 & 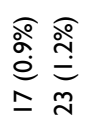 & 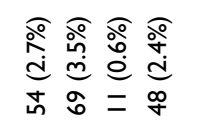 & 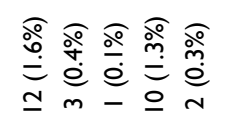 & 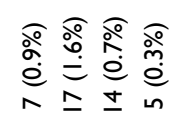 & $\frac{\stackrel{\nwarrow o}{\doteq}}{\bar{\sim}}$ & 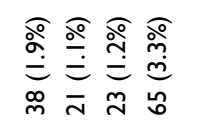 \\
\hline & 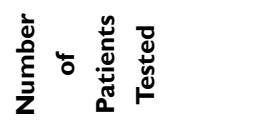 & 妾 & $\underline{\underline{\alpha}}$ & $\underset{\underline{\alpha}}{\widetilde{a}}$ & 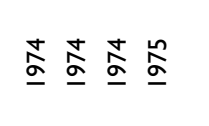 & 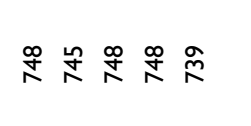 & 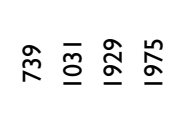 & $\underset{⿱ 亠 幺}{\sigma}$ & 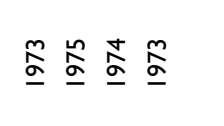 \\
\hline \multirow{5}{*}{ 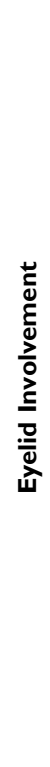 } & 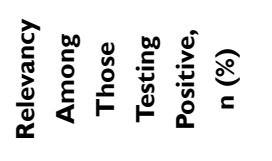 & $\begin{array}{l}\text { ळo } \\
\stackrel{\circ}{\circ} \\
\stackrel{5}{=}\end{array}$ & 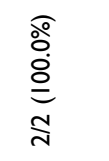 & 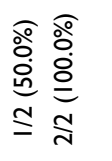 & 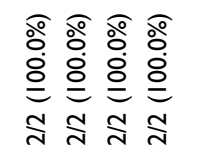 & 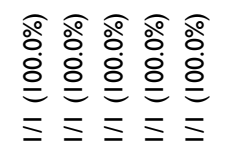 & 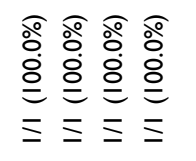 & $\begin{array}{l}\widehat{\circ} \\
\stackrel{\circ}{\circ} \\
\equiv\end{array}$ & 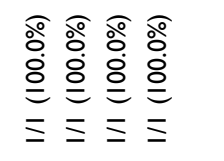 \\
\hline & 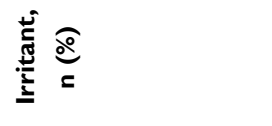 & 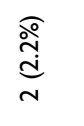 & 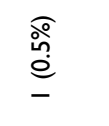 & 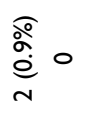 & 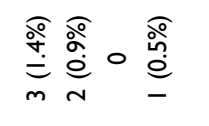 & 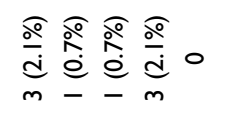 & 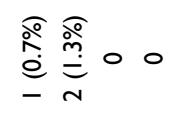 & ํํำ & 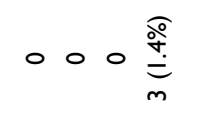 \\
\hline & 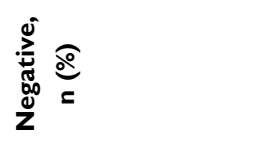 & $\begin{array}{l}\widehat{\circ} \\
\stackrel{0}{0} \\
o \\
\infty\end{array}$ & 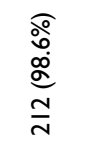 & 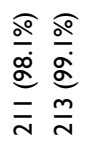 & 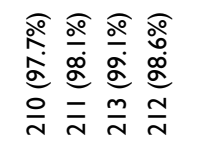 & 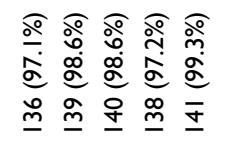 & 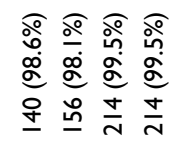 & $\frac{\widehat{o}}{\frac{o}{\sigma}}$ & 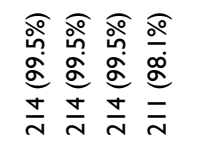 \\
\hline & $\stackrel{0}{\stackrel{0}{0}}$ & 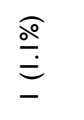 & $\begin{array}{c}\text { @o } \\
\stackrel{2}{2} \\
N\end{array}$ & 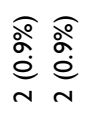 & 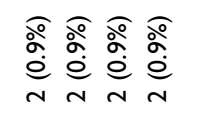 & 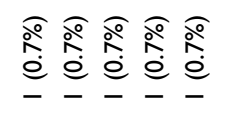 & 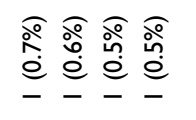 & 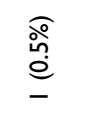 & 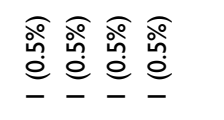 \\
\hline & 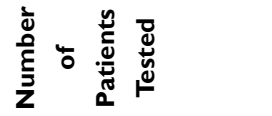 & 8 & $\stackrel{n}{N}$ & $\stackrel{n}{N} \frac{n}{N}$ & $\stackrel{n}{N} \frac{n}{N} \stackrel{n}{N} \frac{n}{N}$ & 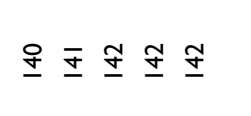 & 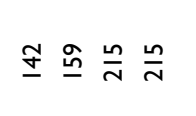 & $\stackrel{n}{N}$ & $\stackrel{n}{N} \frac{n}{N} \frac{n}{N} \frac{n}{N}$ \\
\hline \multicolumn{2}{|c|}{ 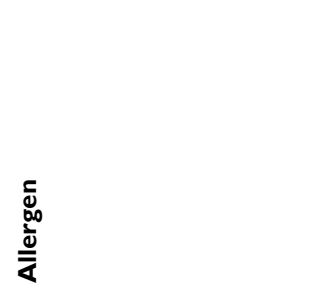 } & 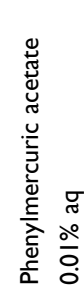 & 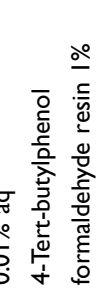 & 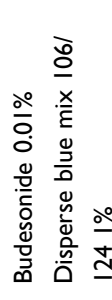 & 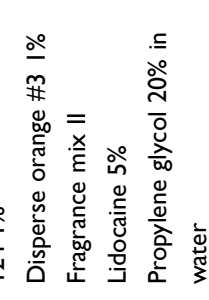 & 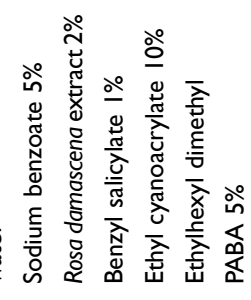 & 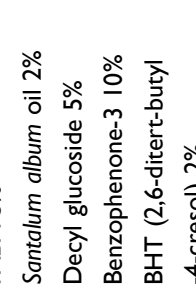 & 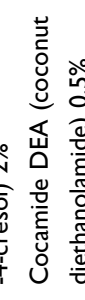 & 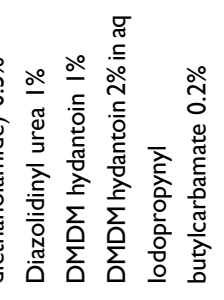 \\
\hline
\end{tabular}




\begin{tabular}{|c|c|c|}
\hline \multicolumn{3}{|c|}{ 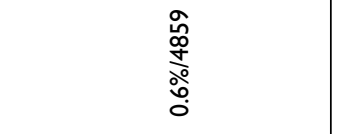 } \\
\hline 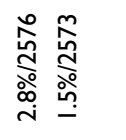 & 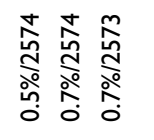 & 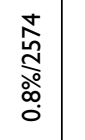 \\
\hline 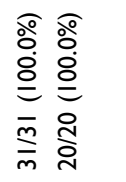 & 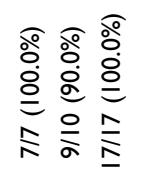 & $\begin{array}{l}\stackrel{\circ}{\circ} \\
\stackrel{\circ}{\circ} \\
\circ \\
\stackrel{\circ}{0}\end{array}$ \\
\hline $\begin{array}{l}\text { oㅇㅇㅇ } \\
\stackrel{\circ}{\stackrel{0}{0}} \\
=\end{array}$ & 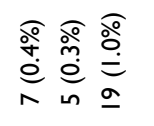 & 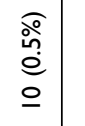 \\
\hline 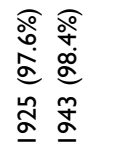 & 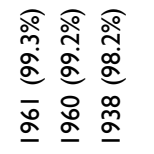 & 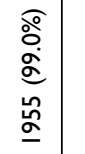 \\
\hline 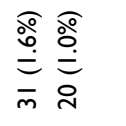 & 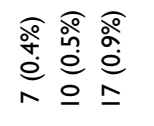 & 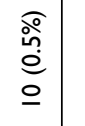 \\
\hline 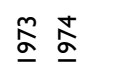 & 疽㑒芯 & $\stackrel{n}{\alpha}$ \\
\hline 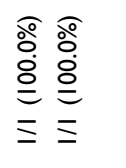 & 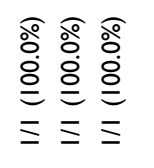 & $\begin{array}{l}\widehat{\circ} \\
\stackrel{\circ}{\circ} \\
\vdots \\
\vdots\end{array}$ \\
\hline 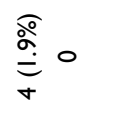 & 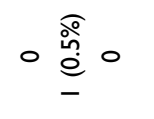 & $\begin{array}{c}\stackrel{\widehat{o}}{\hat{\circ}} \\
\stackrel{\hat{2}}{-}\end{array}$ \\
\hline 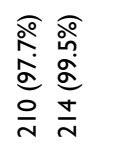 & 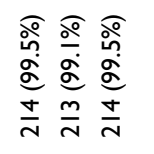 & $\begin{array}{c}\frac{\sigma}{\sigma o} \\
\frac{m}{N} \\
\frac{m}{N}\end{array}$ \\
\hline 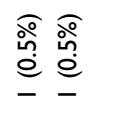 & 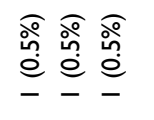 & 突 \\
\hline$\frac{n}{N} \frac{n}{N}$ & $\stackrel{n}{N} \frac{n}{N} \frac{n}{N}$ & $\stackrel{n}{N}$ \\
\hline 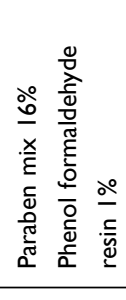 & 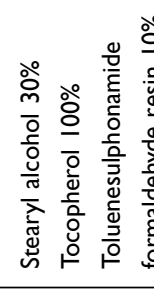 & 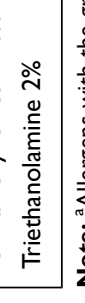 \\
\hline
\end{tabular}

fragrances (in cosmetic and cleansing products); acrylates (in artificial/gel nails); and surfactants (eg in tear-free shampoos).

The Mayo Clinic Standard Series tests the following topical steroids: tixocortol pivalate, hydrocortisone-17butyrate, budesonide, clobetasol-17-butyrate, triamcinolone, and desoximetasone. In the 215 patients with eyelid involvement, the corticosteroids eliciting positive reactions were budesonide and tixocortol pivalate (Group 1 corticosteroids). In the 1975 patients without eyelid involvement, the corticosteroids eliciting positive reactions were tixocortol pivalate, budesonide, hydrocortisone-17butyrate, clobetasol-17-butyrate, and triamcinolone. As shown in Table 4, desoximetasone (a Group 3 corticosteroid) elicited no positive reactions in patients either with or without eyelid involvement.

\section{Discussion}

\section{Allergic Contact Dermatitis with Eyelid Involvement}

For ACD patients with eyelid dermatitis, the seven most common contact allergen groups were identified as follows (in descending order): metals; shellac; preservatives; topical antibiotics; fragrances; acrylates; and surfactants.

For a considerable number of allergens, reaction rates in patients with eyelid involvement were lower than those in patients without eyelid involvement and lower than those in general patch test populations, as shown in Table 2. For example, positive reaction rates to fragrance mix and to a broad palate of preservatives (eg iodopropynyl butylcarbamate) were lower in patients with eyelid involvement. These findings might call into question the commonly offered clinical explanation of the thin eyelid epithelium's particular susceptibility to $\mathrm{ACD} .^{6-12}$ However, patients with eyelid involvement did show higher reaction rates to a few allergens, ie shellac, BAK, acrylates, and surfactants.

Of note, the relevance rates for patch test data shown in Table 2 are high in this study, reflecting a select population of patch test patients where the dermatologist's clinical index of suspicion for contact dermatitis was high.

Nonetheless, patients with eyelid dermatitis in this study were not necessarily diagnosed with dermatitis limited only to the eyelids. Future studies conducted on larger populations might limit diagnoses to eyelid skin involvement only. 
Table 3 Top 15 Allergens Eliciting the Highest Reaction Rates in the Following Four Populations: Mayo Clinic 2013-2017 with Eyelid Involvement, Mayo Clinic 20I3-2017 without Eyelid Involvement, NACDG 20I3-20I4, and MCCDG 20II-20I5 General Patch Test Populations (Sorted by Descending Positivity)

\begin{tabular}{|c|c|c|c|c|}
\hline Rank & $\begin{array}{l}\text { Mayo Clinic 2013-2017 with } \\
\text { Eyelid Involvement }\end{array}$ & $\begin{array}{l}\text { Mayo Clinic 20 I3-20 I7 without } \\
\text { Eyelid Involvement }\end{array}$ & NACDG 2013-2014 & MCCDG 20II-20I5 \\
\hline 1 & Nickel (II) sulfate hexahydrate $2.5 \%$ & Nickel (II) sulfate hexahydrate $2.5 \%$ & Nickel sulfate, $2.5 \%$ pet & Gold sodium thiosulfate, $2 \%$ \\
\hline 2 & Shellac $20 \%$ in alc. & Methylisothiazolinone $0.2 \% \mathrm{aq}^{\mathrm{a}}$ & Fragrance mix I, 8\% pet & Nickel sulfate hexahydrate, $2.5 \%$ \\
\hline 3 & Thimerosal $0.1 \%$ & Myroxylon pereirae resin $25 \%$ & MI, $0.2 \%$ aq & Myroxylon pereirae resin, $25 \%$ \\
\hline 4 & Cobalt (II) chloride hexahydrate I\% & Thimerosal $0.1 \%$ & Neomycin, $20 \%$ pet & $\begin{array}{l}\text { Gold (I) sodium thiosulfate } \\
\text { dehydrate, } 0.5 \%\end{array}$ \\
\hline 5 & Neomycin sulfate $20 \%$ & Shellac $20 \%$ in alc ${ }^{\mathrm{a}}$ & Bacitracin, $20 \%$ pet & $\begin{array}{l}\text { Methylisothiazolinone, } 0.2 \% \\
\text { aqueous }\end{array}$ \\
\hline 6 & $\begin{array}{l}\text { Gold (I) sodium thiosulfate } \\
\text { dihydrate } 0.5 \% \text { in pet }\end{array}$ & Cobalt (II) chloride hexahydrate I\% & Cobalt chloride, $1 \%$ pet & $\begin{array}{l}\text { Benzalkonium chloride, } 0.1 \% \\
\text { aqueous }\end{array}$ \\
\hline 7 & Methylisothiazolinone $0.2 \%$ aq & Neomycin sulfate $20 \%$ & Myroxylon pereirae, $25 \%$ pet & $\begin{array}{l}\text { Cobalt (II) chloride } \\
\text { hexahydrate, } 1 \%\end{array}$ \\
\hline 8 & Myroxylon pereirae resin $25 \%$ & $\begin{array}{l}\text { Gold (I) sodium thiosulfate } \\
\text { dihydrate } 0.5 \% \text { in pet }\end{array}$ & p-Phenylenediamine, I\% pet & Fragrance mix, $8 \%$ \\
\hline 9 & Bacitracin $20 \%$ & Fragrance mix $8 \%^{\mathrm{a}, \mathrm{b}}$ & Formaldehyde, $2 \%$ aq & Neomycin sulfate, $20 \%$ \\
\hline 10 & Potassium dichromate $0.25 \%$ & Potassium dichromate $0.25 \%$ & $\mathrm{MCl} / \mathrm{MI}, 0.01 \%$ aq & Potassium dichromate, $0.25 \%$ \\
\hline 11 & $\begin{array}{l}\text { 2-Hydroxyethyl methacrylate } 2 \% \\
\text { (HEMA) }\end{array}$ & Hydroperoxides of linalool $1.0 \%^{b}$ & Fragrance mix II, $14 \%$ pet & Carba mix, $3 \%$ \\
\hline 12 & Benzalkonium chloride $0.1 \%$ aq & $\mathrm{MCl} / \mathrm{MI} 100 \mathrm{ppm}$ aq & Formaldehyde, $1 \%$ aq & Benzoic acid, $5 \%$ \\
\hline 13 & $\mathrm{MCl} / \mathrm{Ml} 100 \mathrm{ppm}$ aq & Bacitracin $20 \%$ & $\begin{array}{l}\text { Lanolin alcohol (Amerchol } \\
\text { L I0I), } 50 \% \text { pet }\end{array}$ & Propolis, $10 \%$ \\
\hline 14 & Propolis $10 \%$ & Methyldibromo glutaronitrile $0.5 \%$ a, b & Carba mix, $3 \%$ pet & $\begin{array}{l}\text { Methyldibromo glutaronitrile/ } \\
\text { phenoxyethanol, I.5\% }\end{array}$ \\
\hline 15 & Hydroperoxides of linalool $1.0 \%$ & Carba mix $3 \%^{\mathrm{a}, \mathrm{b}}$ & Quaternium-15, 2\% pet & Bacitracin, $20 \%$ \\
\hline
\end{tabular}

Notes: ${ }^{a}$ Allergens with the greatest difference between those with eyelid involvement and without (3.9\% difference or greater). ${ }^{b}$ Allergens in the top I5 without eyelid involvement not found in the top 15 with eyelid involvement.

\section{Allergic Contact Dermatitis without Eyelid Involvement}

Upon comparison of the results from patients with eyelid involvement to those without (using Table 3), it is interesting to note that 11 of the 15 allergens with the highest positive reaction rates were the same across both groups. Since the first five groups (metals, preservatives, fragrances, shellac, and topical antibiotics) are also seen in the top seven groups for patients with eyelid involvement, avoidance of the top allergen groups for patients with eyelid dermatitis also affords promising benefits for the prevention of dermatitis on other areas of the body.

\section{Topical Steroids}

Topical corticosteroids, as shown in Table 1, can be classified into three groups according to Baeck and co-authors: Group 1 comprises non-methylated, most often nonhalogenated molecules; Group 2 comprises halogenated molecules with a $\mathrm{C}_{16} / \mathrm{C}_{17}$ cis ketal/diol structure; and Group 3 comprises halogenated and $\mathrm{C}_{16}$-methylated molecules. ${ }^{23,24}$ Group 1 steroids are the most common offenders in steroid-triggered allergic reactions, while Group 3 steroids are the least common. ${ }^{24}$

The only corticosteroids eliciting positive reactions in ACD patients with eyelid involvement were budesonide and tixocortol pivalate, which are both Group 1 


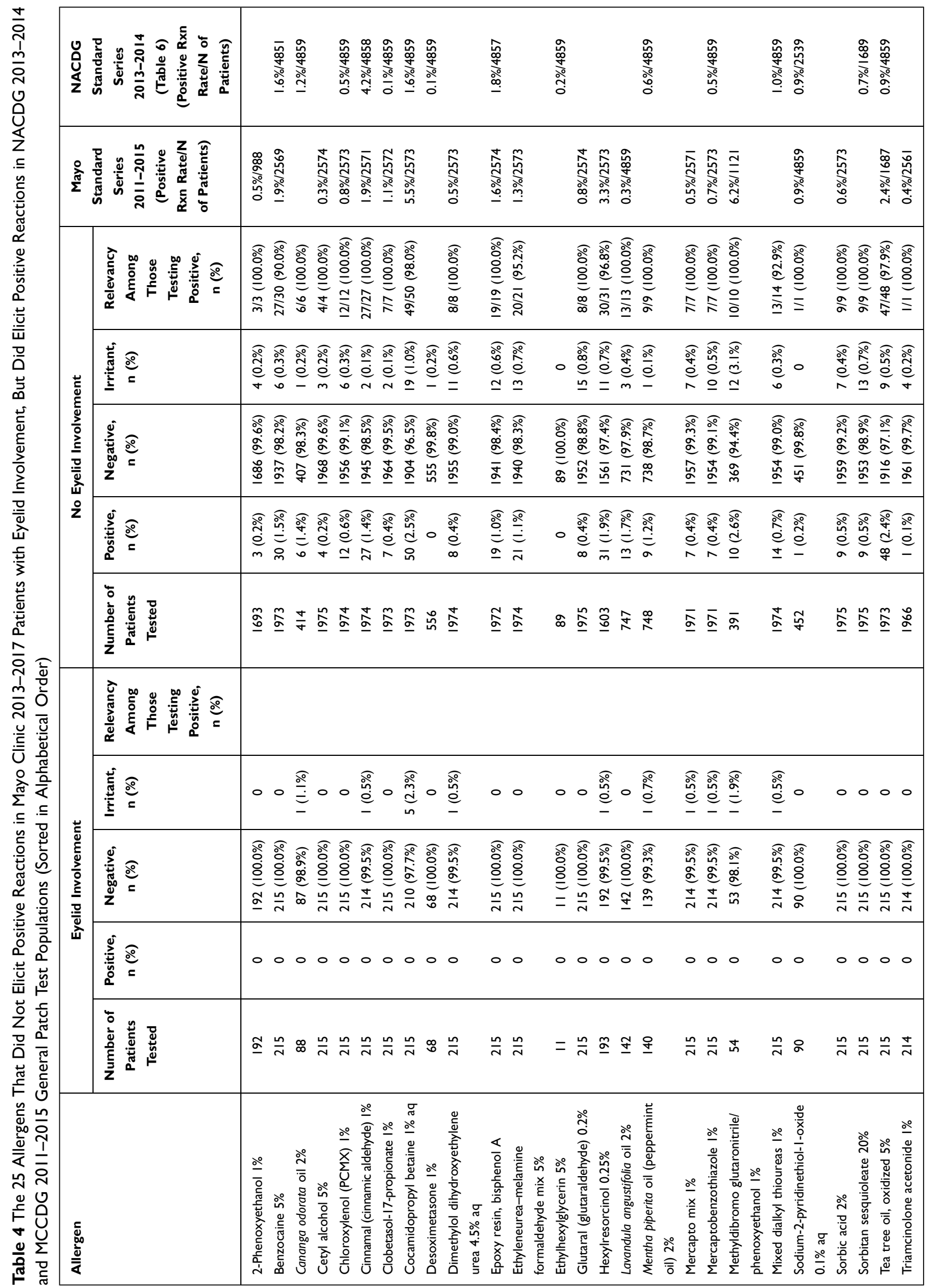


corticosteroids; meanwhile, the Group 3 corticosteroid desoximetasone elicited no positive reactions in ACD patients with or without eyelid involvement.

Although topical steroids did not appear in our top seven allergen groups, they remain of note in discussion, in light of their widespread use in ophthalmology and the literary gap on their paradoxical role as both potential ACD allergen and treatment for allergic conditions. ${ }^{23}$ For patients with ACD involving the eyelids, a switch to Group 3 steroids may offer clinical benefit.

Topical steroids should not be overlooked in patch tests for eyelid dermatitis.,

\section{Ocular Medications}

Out of 365 patient products tested at Mayo Clinic from 2013 to 2017, only 12 elicited positive reaction rates, of which three were eye medications. Eye care providers often use a trial off of all eye medications to look for symptomatic improvement, or a unilateral trial of the suspected agent. Given that specific causal agents of patients' dermatitis within patient products (ie active versus inactive ingredients) cannot be ascertained, the ideal management of patients presenting with yet unexplained dermatitis involves patch testing of patients' own eye medications and close examination of ingredients to test true offenders not included in standard series. ${ }^{13}$ For instance, BAK is still the most commonly used preservative in ophthalmic preparations, including tetracaine hydrochloride, timolol maleate solution, and brimonidinetimolol combination drops, and it may be the underlying culprit responsible for the positive reactions to these patient products seen in this study.

\section{Metals}

Given the relatively high reaction rates to nickel, cobalt, gold, and chrome found in this study, it is advisable to counsel patients with eyelid dermatitis to optimally avoid costume jewelry, nickel in eyewear (sunglasses or prescription glasses), gold- and chrome-containing cosmetics, nail lacquers, and some skin cleansers and moisturizers, facilitated by the use of an allergen avoidance database.

\section{Nickel and Cobalt}

Nickel has been found in certain makeup products (eg eye shadow, mascara), which may explain the greater incidence of reported nickel sensitivity in female patients. ${ }^{4,9,27}$ Multiple studies across the past decade have found nickel to be among the most common contact allergens, if not the most common. ${ }^{9,26,28}$ A few reports have noted ACD-inducing nickel found in eyelash curlers. ${ }^{4,29}$ Therefore, avoidance of eyelash curlers may also be advisable, because of the potential allergenic composition of their metal frames. Additionally, cobalt exposure has been noted to correlate with nickel exposure. ${ }^{4,27}$ Although nickel is generally not used in contemporary cosmetics, modified cobalt (eg cyanocobalamin and amine cobalt salt) is occasionally used in cosmetics and household products.

\section{Gold}

Sensitivity of many individuals to gold as a contact allergen has been well established in dermatologic literature (though not as widely reported in the ophthalmic literature), and the American Contact Dermatitis Society (ACDS) previously nicknamed it the "allergen of the year." ${ }^{, 411,16,30}$ Indeed, in the NACDG 2003-2004 study, gold was the most common contact allergen responsible for causing dermatitis limited to the eyelids. ${ }^{30}$ Skincare products known to contain gold include eye shadow, mascara, foundations, moisturizers, and eye masks. Outside of the presence of gold in these products or the occasional patient who develops a red rash under gold jewelry, the high rate of gold allergy is often of uncertain clinical significance. A one month trial off of gold jewelry is best reserved for patients with a history of a rash under gold jewelry.

\section{Chrome}

Lastly, the role of chrome in eyelid dermatitis has not been emphasized as a potential eyelid allergen and therefore represents an important finding in our study. Although potassium dichromate was not found in the top 15 contact allergens in the NACDG 2013-2014 study, it was found in those for the MCCDG 2011-2015 study. ${ }^{20,26}$ Of note, 1422 skincare products active in the online allergen avoidance program SkinSAFE (out of 48,017 total products as of April 27, 2020) contain a version of chromates (eg chromium oxide green), used for green hue in numerous makeup products. Potassium dichromate is also used in many household products, eg glues, leather, and polishes. ${ }^{20}$

\section{Shellac}

Products that contain shellac or shellac wax (used as a tackifier in eye makeup products) include mascara, lipstick, and adhesive tooth whitening strips. Shellac, derived from lac beetle secretions, is included in neither the NACDG 2013-2014 Standard Series nor the ACDS 2017 allergen 
Table 5 Products on the Market Containing Thimerosal

\begin{tabular}{|l|l|}
\hline Product Name & Manufacturer \\
\hline Cortisporin Ophthalmic Suspension (RX) $7.5 \mathrm{~mL}$ & Monarch Pharmaceuticals \\
\hline $\begin{array}{l}\text { Neomycin Sulfate/Polymyxin B Sulfate/ } \\
\text { Hydrocortisone Otic Suspension (RX) } 10 \mathrm{~mL}\end{array}$ & Sandoz \\
\hline $\begin{array}{l}\text { Neosporin Ophthalmic Solution Sterile (RX) } \\
10 \mathrm{~mL}\end{array}$ & Monarch Pharmaceuticals \\
\hline $\begin{array}{l}\text { Ocufen 0.03\% Ophthalmic Solution (RX) } 2.5 \mathrm{~mL} \\
\text { Poly-Pred Ophthalmic Suspension (RX) } 5 \mathrm{~mL}\end{array}$ & Allergan \\
\hline Viroptic I\% Ophthalmic Solution (RX) $7.5 \mathrm{~mL}$ & Monarch Pharmaceuticals \\
\hline
\end{tabular}

Note: SkinSAFE database query on February 6, 2021.

panels. ${ }^{31}$ Given its high reaction rate in our data (16.4\%), shellac is important to note for eyelid dermatitis patients.

\section{Preservatives}

In the MCCDG 2011-2015 study, the 15 most common allergens included the preservatives methyldibromo glutaronitrile and BAK. ${ }^{20}$ In this current study, BAK (found in skincare products, prescription and over-the-counter eye preparations) was also included in the 15 most common eyelid ACD allergens; meanwhile, methyldibromo glutaronitrile was included in those for ACD patients without eyelid involvement.

Thimerosal, known for inhibition of bacterial growth in ophthalmic solutions, has developed a reputation as a problematic contact allergen, noted as early as $1988 .{ }^{9,32}$ It is now rarely used in vaccines, eye drop medications, or cleansing products for contact lenses; within the Environmental Working Group personal care product database as of April 27, 2020, only eight products included thimerosal, all of which were labeled "old formulation." Table 5 displays products on the market that contain thimerosal, according to the SkinSAFE database as of February 6, 2021.

Another preservative increasingly reported to elicit positive allergic reactions is methylisothiazolinone (MI), found in hair products, lotions, and shampoos. ${ }^{9,13,20,26}$ In fact, both MI and methylchloroisothiazolinone/methylisothiazolinone (MCI/MI) ranked in the 15 most common contact allergens in this study, for populations both with and without eyelid involvement.

Manufacturers moving away from thimerosal and MI will often shift to BAK (or its derivatives), which also has emerging prevalence as a contact allergen, as seen in this study. ${ }^{20}$ Like thimerosal, it has been employed in ophthalmic products for its antimicrobial properties. ${ }^{8,20,32}$ BAK has been studied and described as "more toxic" than thimerosal, but its presence (or one of its cross-reactors) has been noted in a significant number of products, including many eye prescription and over-the-counter drops, cosmetics, hand sanitizers, deodorants, detergents, shampoos, and liquid soaps. ${ }^{7,8,20}$

\section{Topical Antibiotics}

Eye care providers are well aware of known allergic reactions to antibiotics neomycin and bacitracin, which are inexpensive and commonly used in eye medications. Use of these products should be avoided in patients with eyelid $\mathrm{ACD}^{4}$

\section{Fragrances}

The fragrances in our top 15 most common contact allergens for eyelid dermatitis are (in order of descending positivity) Myroxylon pereirae resin, also known as balsam of Peru; propolis, also known as bee glue; and hydroperoxides of linalool (which imparts the aroma of lavender). Fragrances have a significant amount of crossreactivity and are found in a wide variety of consumer products including soaps, shampoos, perfumes, and cosmetics (eg eye makeup).

\section{Acrylates}

Hydroxyethyl methacrylate (HEMA) was one acrylate in our 15 most common eyelid ACD allergens. Especially in recent years, HEMA has been noted as an allergen of rising prominence; the NACDG 2013-2014 study found a statistically significant increase in its positive reaction rate, compared to both its rate in the past two reporting periods and the pooled rate from the prior 6 years. ${ }^{26}$ HEMA and other acrylates, as components of nail polish, artificial and gel nails, are known contributors to eyelid dermatitis (due to allergen transfer to the eyelid), sometimes also presenting with characteristic nailbed erythema representing periungual dermatitis. ${ }^{4,8}$ Eliciting a history of artificial nail application and discussing discontinuation of this product as appropriate may be helpful for ACD patients.

Some acrylates, such as polymethyl methacrylate found in intraocular lenses and fluorosilicone acrylates in rigid contact lenses, are known to be well tolerated by the eye. For patients allergic to acrylates, it is important to clarify that although contact lenses are made of acrylates, 
they are made of "cured" (polymerized) acrylates, and therefore do not pose a risk of contact allergy.

\section{Surfactants}

Surfactants such as oleamidopropyl dimethylamine and amidoamine can be found in products such as tear-free, baby shampoos used for seborrheic blepharitis. Cocamidopropyl betaine, another surfactant, can be found in liquid soaps, shampoos, and eyelid hygiene products, which may be frequently used in ophthalmology clinics. $^{21,33}$ Although less well recognized, cocamide diethanolamine, derived from coconut oil with diethanolamine, has also been reported to cause eyelid dermatitis. ${ }^{34}$ The role of these allergens in ACD has been well established in dermatologic journals since the 1980s, but not yet so in ophthalmologic journals..$^{21,33}$

\section{Eyelid ACD Management}

Accurate identification and proper avoidance of problematic allergens offers the most straightforward, effective cure for eyelid ACD, as continued rubbing and itching can worsen symptoms. ${ }^{1,2,17-19}$ Patch testing is widely regarded as the ACD gold standard, but is not always readily available. ${ }^{17-19}$ Even without it, patients can be instructed to access free, online allergen avoidance programs to find safe products, such as SkinSAFE [www.SkinSafeProducts.

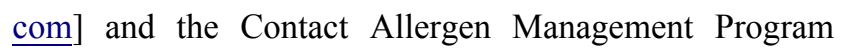
(CAMP) [www.ContactDerm.org]. Specifically, health care providers can enter the top seven allergen groups into CAMP (with ACDS member registration), or patients can browse SkinSAFE "EyelidSAFE" products, to choose products (both prescription and over-the-counter) free of these ACD triggers.

From an eye care provider's prescribing perspective, ophthalmic products can contain three common categories of allergens: preservatives, antibiotics, and topical steroids. Therefore, empiric common allergen avoidance therapy includes prescription of ophthalmic products 1) free of the preservative BAK; 2) free of aminoglycoside antibiotics (neomycin, gentamycin, tobramycin) and bacitracin; and 3) although less commonly a source for contact allergy, free of steroids such as prednisolone, loteprednol, and fluorometholone, with preference for dexamethasone or rimexolone. From a cutaneous therapy perspective, cold compresses, antihistamines, and topical corticosteroids (such as dexamethasone, given its low rate of allergy) can be employed to reduce patient discomfort and, in the case of topical corticosteroids, expedite resolution of acute inflammation. ${ }^{1,4,6,16,35}$ When using topical ophthalmic or cutaneous eyelid corticosteroids, the lowest-dose, shortest course is recommended, given the potential for steroid addiction and side effects. Long-term use can result in eyelid skin atrophy, cataracts, glaucoma, tachyphylaxis, or even flaring erythema, so eye examinations and intraocular pressure monitoring are needed. ${ }^{1,6,36}$ Local compounding pharmacies can make preservative-free dexamethasone and other ophthalmic medications to assist patients reacting to commercially prepared drops with BAK. If patients continue to flare upon tapering topical steroids, despite empiric top common allergen avoidance, chronic topical immunosuppressive options to be considered include steroid-sparing calcineurin inhibitors (eg tacrolimus and pimecrolimus) and the novel non-steroidal anti-inflammatory phosphodiesterase-4 inhibitor crisaborole. ${ }^{37-39}$ For eyelids, tacrolimus is the calcineurin inhibitor of choice, as pimecrolimus is currently only available as a cream containing the moderately frequent contact allergen propylene glycol. Of note, crisaborole (Eucrisa) also contains propylene glycol. Additionally, if a patient requires chronic topical immunosuppression, patch testing should be considered.

Nonetheless, this study's findings and recommendations may be limited in the following ways: its retrospective nature precludes an evaluation for the efficacy of empiric avoidance of the top seven allergen groups; it does not address allergic conjunctivitis and dry eye syndrome (which can manifest as contact dermatitis); and its relatively small sample of 215 patients with periorbital dermatitis, patch tested at Mayo Clinic from 2013 to 2017, mostly comprised Caucasian and female patients, who are not fully representative of general patient populations. Finally, these top allergen groups discussed are not exhaustive, and are subject to change as products evolve and patients develop new sensitivities.

\section{Conclusions}

The top seven most common allergen groups involved in eyelid ACD are metals, shellac, preservatives (eg, BAK), topical antibiotics (eg, aminoglycosides and bacitracin), fragrances, acrylates, and surfactants (eg, cocamidopropyl betaine). This finding is in line with previous studies, which identify topical eye medications, cosmetics, hair products, preservatives, antibiotics, nail products, and facial skincare products as eyelid ACD triggers. ${ }^{6-8,12,16,22}$

Interpreting patient product labels with the most common contact allergens is not straightforward, primarily 
owing to the complexity of ingredient names, synonyms, or allergenic cross-reactors. Moreover, cosmetic product labeling does not require Food and Drug Administration (FDA) premarket approval; companies are responsible for ensuring compliance with all FDA labeling requirements. Online allergen avoidance programs save time that might otherwise be spent manually searching product labeling for specific allergen-free brands. These programs support patient empowerment and self-sufficiency on the journey to avoid common offending allergens and prevent ACD recurrence.

Ophthalmologists and optometrists are the first-line providers to evaluate and treat eyelid ACD. Sometimes patient use of a product over the entire body may manifest only as eyelid dermatitis. Understanding ACD diagnostic and treatment strategies is important for the improvement of patient outcomes. When simple product avoidance fails to alleviate $\mathrm{ACD}$, patch testing to identify allergenic ingredients is recommended, in conjunction with online databases for safe products free of these problematic agents. For patients without access to patch testing, education about the top seven allergen groups and avoidance of products with these ingredients can be helpful. We acknowledge that empiric avoidance of all top common allergens may represent a clinical burden, and we therefore recommend avoidance only if clinical suspicion of contact dermatitis is high. Lastly, for patients suspected of allergy to the most commonly used topical steroid eye drops, providers should choose Group 3 steroids, such as dexamethasone or rimexolone, or even preservative-free compounded dexamethasone.

\section{Acknowledgments}

The abstract of this paper was presented at the 2019 Association for Research in Vision and Ophthalmology Annual Meeting as a poster presentation. The poster's abstract was published in 'Poster Abstracts' in Investigative Ophthalmology \& Visual Science: https:// iovs.arvojournals.org/article.aspx?articleid=2746133.

\section{Funding}

No grants or other sources of financial support were necessary for this retrospective chart review. J.F. Shen receives research funding from Ocugen Pharmaceuticals. No federal or industry funding was used for this study.

\section{Disclosure}

J.A. Yiannias is the creator of and consultant for the skin allergy management program SkinSAFE, formerly known as CARD (Contact Allergen Replacement Database). For the remaining authors, there are no conflicts of interest to disclose.

\section{References}

1. Zug KA, Palay DA, Rock B. Dermatologic diagnosis and treatment of itchy red eyelids. Surv Ophthalmol. 1996;40(4):293-306. doi:10.1016/S0039-6257(96)82004-2

2. Grey KR, Warshaw EM. Allergic contact dermatitis to ophthalmic medications: relevant allergens and alternative testing methods. Dermatitis. 2016;27(6):333-347. doi:10.1097/DER.0000000000000224

3. Morris S, Barlow R, Selva D, Malhotra R. Allergic contact dermatitis: a case series and review for the ophthalmologist. $\mathrm{Br}$ J Ophthalmol. 2011;95(7):903-908. doi:10.1136/bjo.2009.174607

4. Guin JD. Eyelid dermatitis: experience in 203 cases. $J$ Am Acad Dermatol. 2002;47(5):755-765. doi:10.1067/mjd.2002.122736

5. Katayama I, Koyano T, Nishioka K. Prevalence of eyelid dermatitis in primary Sjogren's syndrome. Int J Dermatol. 1994;33(6):421-424. doi:10.1111/j.1365-4362.1994.tb04044.x

6. Papier A, Tuttle DJ, Mahar TJ. Differential diagnosis of the swollen red eyelid. Am Fam Physician. 2007;76(12):1815-1824.

7. Wolf R, Orion E, Tuzun Y. Periorbital (eyelid) dermatides. Clin Dermatol. 2014;32(1):131-140. doi:10.1016/j.clindermatol.2013.05.035

8. Amin KA, Belsito DV. The aetiology of eyelid dermatitis: a 10-year retrospective analysis. Contact Dermatitis. 2006;55(5):280-285. doi:10.1111/j.1600-0536.2006.00927.x

9. Herro EM, Elsaie ML, Nijhawan RI, Jacob SE. Recommendations for a screening series for allergic contact eyelid dermatitis. Dermatitis. 2012;23(1):17-21. doi:10.1097/DER.0b013e31823d191f

10. Katoh N, Murakami M, Yasuno H. Eyelid dermatitis caused by delayed-type hypersensitivity to natural rubber latex. Contact Dermatitis. 1999;40(6):336-337. doi:10.1111/j.1600-0536.1999.tb06 095.x

11. McDaniel LM, Couch SM. Allergic eyelid dermatitis as the sole manifestation of gold hypersensitivity. Ophthalmic Plast Reconstr Surg. 2017;33(4):e80-e82. doi:10.1097/IOP.0000000000000783

12. Nethercott JR, Nield G, Holness DL. A review of 79 cases of eyelid dermatitis. J Am Acad Dermatol. 1989;21(2):223-230. doi:10.1016/ S0190-9622(89)70165-1

13. Assier H, Tetart F, Avenel-Audran M, et al. Is a specific eyelid patch test series useful? Results of a French prospective study. Contact Dermatitis. 2018;79(3):157-161. doi:10.1111/cod.13040

14. Jacob SE, Stechschulte S. Eyelid dermatitis associated with balsam of Peru constituents: benzoic acid and benzyl alcohol. Contact Dermatitis. 2008;58(2):111-112. doi:10.1111/j.1600-0536.2007.01178.x

15. Valsecchi R, Imberti G, Martino D, Cainelli T. Eyelid dermatitis: an evaluation of 150 patients. Contact Dermatitis. 1992;27(3):143-147. doi:10.1111/j.1600-0536.1992.tb05242.x

16. Chisholm SAM, Couch SM, Custer PL. Etiology and management of allergic eyelid dermatitis. Ophthalmic Plast Reconstr Surg. 2017;33 (4):248-250. doi:10.1097/IOP.0000000000000723

17. Beltrani VS, Beltrani VP. Contact dermatitis. Ann Allergy Asthma Immunol. 1997;78(2):160-166. doi:10.1016/S1081-1206(10)63383-2

18. Chaudhry HM, Drage LA, El-Azhary RA, et al. Delayed patch-test reading after 5 days: an update from the Mayo Clinic contact dermatitis group. Dermatitis. 2017;28(4):253-260. doi:10.1097/DER.00000 00000000297 
19. Zhu TH, Suresh R, Warshaw E, et al. The medical necessity of comprehensive patch testing. Dermatitis. 2018;29(3):107-111. doi:10.1097/DER.0000000000000362

20. Veverka KK, Hall MR, Yiannias JA, et al. Trends in patch testing with the Mayo Clinic standard series, 2011-2015. Dermatitis. 2018;29(6):310-315. doi:10.1097/DER.0000000000000411

21. Welling JD, Mauger TF, Schoenfield LR, Hendershot AJ. Chronic eyelid dermatitis secondary to cocamidopropyl betaine allergy in a patient using baby shampoo eyelid scrubs. JAMA Ophthalmol. 2014;132(3):357-359. doi:10.1001/jamaophthalmol.2013.6254

22. Novitskaya ES, Dean SJ, Craig JP, Alexandroff AB. Current dilemmas and controversies in allergic contact dermatitis to ophthalmic medications. Clin Dermatol. 2011;29(3):295-299. doi:10.1016/j. clindermatol.2010.11.008

23. Baeck M, Chemelle JA, Goossens A, Nicolas JF, Terreux R. Corticosteroid cross-reactivity: clinical and molecular modelling tools. Allergy. 2011;66(10):1367-1374. doi:10.1111/j.1398-9995.2011.02666.x

24. Baeck M, Goossens A. Immediate and delayed allergic hypersensitivity to corticosteroids: practical guidelines. Contact Dermatitis. 2012;66(1):38-45. doi:10.1111/j.1600-0536.2011.01967.x

25. Davis MD, Scalf LA, Yiannias JA, et al. Changing trends and allergens in the patch test standard series: a mayo clinic 5-year retrospective review, january 1, 2001, through december 31, 2005. Arch Dermatol. 2008;144(1):67-72. doi:10.1001/archdermatol.2007.2

26. DeKoven JG, Warshaw EM, Belsito DV, et al. North American contact dermatitis group patch test results 2013-2014. Dermatitis. 2017;28(1):33-46. doi:10.1097/DER.0000000000000225

27. Shah M, Lewis FM, Gawkrodger DJ. Facial dermatitis and eyelid dermatitis: a comparison of patch test results and final diagnoses. Contact Dermatitis. 1996;34(2):140-141. doi:10.1111/j.1600-0536. 1996.tb02148.x

28. Crouse L, Ziemer C, Lugo-Somolinos A. Trends in eyelid dermatitis. Dermatitis. 2018;29(2):96-97. doi:10.1097/DER.0000000000000338

29. Henke U, Boehncke WH. Eyelid dermatitis caused by an eyelash former. Contact Dermatitis. 2005;53(4):237. doi:10.1111/j.0105-18 73.2005.0670d.x
30. Rietschel RL, Warshaw EM, Sasseville D, et al. Common contact allergens associated with eyelid dermatitis: data from the North American contact dermatitis group 2003-2004 study period. Dermatitis. 2007;18(2):78-81. doi:10.2310/6620.2007.06041

31. Schalock PC, Dunnick CA, Nedorost S, Brod B, Warshaw E, Mowad C. American contact dermatitis society core allergen series: 2017 update. Dermatitis. 2017;28(2):141-143. doi:10.1097/DER.000 0000000000261

32. Tosti A, Tosti G. Thimerosal: a hidden allergen in ophthalmology. Contact Dermatitis. 1988;18(5):268-273. doi:10.1111/j.1600-0536. 1988.tb02831.x

33. Knopp E, Watsky K. Eyelid dermatitis: contact allergy to 3-(dimethylamino)propylamine. Dermatitis. 2008;19(6):328-333. doi:10. 2310/6620.2008.07071

34. Dejobert Y, Delaporte E, Piette F, Thomas P. Eyelid dermatitis with positive patch test to coconut diethanolamide. Contact Dermatitis. 2005;52(3):173. doi:10.1111/j.0105-1873.2005.0548n.x

35. Guin JD. Eyelid dermatitis: a report of 215 patients. Contact Dermatitis. 2004;50(2):87-90. doi:10.1111/j.0105-1873.2004.00311.x

36. Rapaport MJ, Rapaport V. Eyelid dermatitis to red face syndrome to cure: clinical experience in 100 cases. J Am Acad Dermatol. 1999;41 (3):435-442. doi:10.1016/S0190-9622(99)70118-0

37. Hoy SM. Crisaborole ointment $2 \%$ : a review in mild to moderate atopic dermatitis. Am J Clin Dermatol. 2017;18(6):837-843. doi:10.1007/s40257-017-0327-4

38. Katsarou A, Armenaka M, Vosynioti V, Lagogianni E, Kalogeromitros D, Katsambas A. Tacrolimus ointment $0.1 \%$ in the treatment of allergic contact eyelid dermatitis. J Eur Acad Dermatol Venereol. 2009;23(4):382-387. doi:10.1111/j.1468-3083.2008.03056.x

39. Ladda M, Sandhu V, Ighani A, Yeung J. Off-label uses of topical pimecrolimus. J Cutan Med Surg. 2019;23(4):442-448. doi:10.1177/ 1203475419847950
Clinical Ophthalmology

\section{Publish your work in this journal}

Clinical Ophthalmology is an international, peer-reviewed journal covering all subspecialties within ophthalmology. Key topics include: Optometry; Visual science; Pharmacology and drug therapy in eye diseases; Basic Sciences; Primary and Secondary eye care; Patient Safety and Quality of Care Improvements. This journal is indexed on PubMed

\section{Dovepress}

Central and CAS, and is the official journal of The Society of Clinical Ophthalmology (SCO). The manuscript management system is completely online and includes a very quick and fair peer-review system, which is all easy to use. Visit http://www.dovepress.com/ testimonials.php to read real quotes from published authors. 